\title{
Design of an electrical drive for motorized bicycles
}

\author{
C. Boccaletti, G. Duni, P. Petrucci, E. Santini \\ Department of Electrical Engineering \\ University of Rome "La Sapienza" \\ Via Eudossiana 18, 00184 Rome, Italy \\ Tel. +3906 44585762, Fax +39064883235 \\ e-mail: chiara.boccaletti@uniroma1.it
}

\begin{abstract}
The aim of this work is the design of an electrical drive for a motorized bicycle, characterised by new solutions for the control method and the regenerative braking system. A dynamic model of the vehicle has been realised, and the characteristics of the drive have been individuated. An effective closed-loop control strategy has been studied, adjusting the motor torque and the current in order to increase the availability range. Finally, a feasibility study of a regenerative braking system based on the supercapacitor technology has been carried out. All the components of the drive have been selected among the models available on the market. In the paper the results of the simulations are presented and other technical-economical aspects such as energy consumption and costs are also briefly discussed.
\end{abstract}

\section{Key words}

Power-assisted bicycle, radial flux permanent magnet DC motor, supercapacitors, regenerative power control

\section{Introduction}

Human-powered hybrid electric vehicles can become a key to personal transportation in an environment where atmospheric pollution must be limited, where automotive traffic congestion is severe, and where parking space in urban centres is not available [1].

According to the European Law 2002/24/CE item h point 1 , a power-assisted bicycle is a two or three wheels vehicle equipped with an auxiliary electric motor whose maximum nominal power is $0.25 \mathrm{~kW}$.

Electrical bicycles offer extremely efficient, pollutionfree transportation for urban and suburban areas, and the addition of electric drive extends their range.

Motorized bicycles are an economic and ecological vehicle suitable for all ages; the use of a helmet is not compulsory; they will not normally require registration and taxes, licensing or operator qualification [2, 3].

In this paper, the term "motorized bicycle" is used to describe a partially motor powered bicycle, commonly known as "pedelec" (Pedal electric cycle) [3].

The motor action is progressively reduced and finally interrupted if a $25 \mathrm{~km} / \mathrm{h}$ speed is reached (such speed limit is imposed for security reasons), if the cyclist stops pedalling or if the brake is used. Pedalling is the main form of propulsion, while the motor gives extra speed, especially uphill.
The electrical drive consists of four main components:

1) a motor

2) a power transmission system

3) a control system

4) a battery pack

The battery pack and thus the vehicle autonomy is the main aspect to be focused on. In this sense, a closed loop control circuit for the output power control has been studied to be implemented in the electrical drive, avoiding undesired accelerations and increasing the battery range. Such a solution is not commercially available. The recovering of the braking energy by means of the supercapacitor technology can determine a reduction of the electromagnetic stresses on the battery pack, and therefore a longer battery life.

\section{Electrical Drive Design}

The power propelling a bicycle and rider goes mostly into overcoming wind resistance and lifting mass up hills at normal bicycle speeds [2, 3].

Bearing and tire friction are small but can equal wind resistance at very low speeds.

The electric motor torque curve is a function of road slope $\mathrm{p}$, rolling friction coefficient $\mathrm{C}_{\text {roll }}$ (whose value depends on the road conditions), wind speed $V_{w}$, cyclist resistance coefficient $C_{r}$, and total mass $m$ of the bicyclecyclist system. Equation (1) represents the equilibrium.

$$
T_{\text {tot }}=T_{\text {air }}+T_{\text {slope }}+T_{\text {friction }}
$$

Fig. 1 shows the influence of the total mass of the system on the required power.

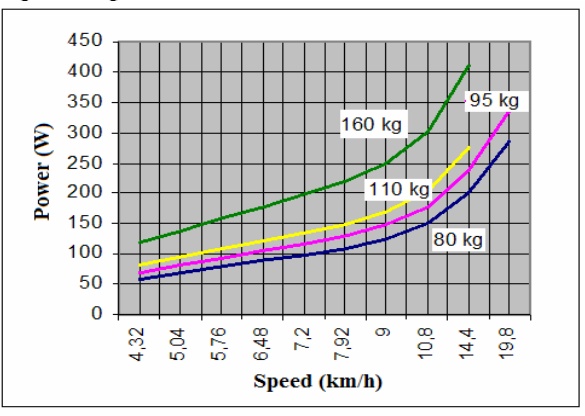

Fig. 1. - Mass influence on the total required power 


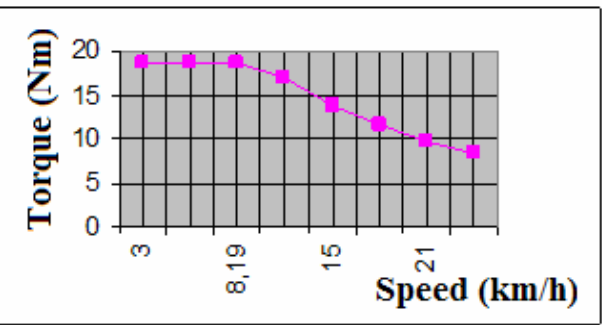

Fig. 2. - Operating limit for the DC brushless machine

A constant value of phase current is imposed; such value remains constant until a speed value $n_{n}$ is reached. It is possible to estimate the electrical power required by the motor. For the present case a limit $\mathrm{P}_{\mathrm{n}}=250 \mathrm{~W}$ is fixed by the law, corresponding to speed $\mathrm{n}_{\mathrm{n}}$.

Therefore, the operation is limited by a curve in which electromagnetic torque and speed change in a way that the power absorbed by the motor is constant (see Fig. 2).

\section{Control System}

To optimise the drive operation, the electromagnetic torque should be independent from the angular position of the rotor. In other words, the phase difference between the magnetic field produced by the permanent magnets and that produced by the stator currents should be maintained equal to $90^{\circ}$. A particular current commutation sequence has to be imposed.

The presence of the three Hall-effect sensors allows to know the instantaneous rotor position and to perform a correct phase current commutation.

The regulator output voltage signal is sent to and compared with the constant frequency triangular signal, in order to generate the static switch command signals of the power inverter.

By means of the studied closed loop control system, for each load condition the motor supplies the correct torque, depending on the level of assistance required, avoiding unwanted accelerations with a consequent improvement of the battery autonomy.

\section{Regenerative Braking}

The battery types chosen in this work are Nickel Metal Hydrate with a nominal voltage of $36 \mathrm{~V}$ and a 9Ah capacity.

Since the level of assistance is strongly influenced by the battery range, the management of the battery charge and discharge phases is particularly important.

The possibility to recover the braking energy is of great interest in designing the electrical drive. The regenerative power control for electric bicycle method is a simple and a low-cost solution [5].

Under appropriate conditions, the batteries can be recharged. During a deceleration or braking, an amount of kinetic energy is usually lost as friction on the wheel. The regenerative braking system allows recovering part of such kinetic energy, to be used to feed either the battery or the electrical drive.

The intermittent characteristic of the itinerary lead by the rider can be smoothed by the introduction of a supercapacitor bank. As it is known, a supercapacitor can store amounts of energy and then distribute it depending on the required power, minimising the energy losses.

The core of the system is represented by a Buck-Boost converter with IGBT power static switches. The Boost side is connected to the supercapacitors bank, the Buck side to the battery pack.

The control system measures the following quantities: the battery and the supercapacitors bank voltages, the state of charge of the battery, the bicycle speed, and the instantaneous currents on the load and on the supercapacitors bank. A microcontroller elaborates those quantities and generates a commutation sequence by means of the PMW technique to control the power static switch.

With the above technical solutions, a performance improvement up to $15-20 \%$ can be obtained.

\section{Conclusions}

A number of different aspects thrust the use of electric bicycles in different situations. These include lower energy cost per distance travelled for a single rider, savings in other costs such as insurances, licenses, registration, parking, improvement of the traffic flow, environmental friendliness, and the health benefit for the rider.

In the paper, the design of an electrical drive for a motorised bicycle is described, using commercial components available on the market.

A dynamical model has been used to simulate the system behaviour in a number of different situations. A closedloop control circuit allows the optimisation of the component operation, determining in particular a proper value of the motor torque with respect to the load and of the absorbed current. In this way, undesired accelerations can be avoided and the battery range can be increased.

Also a suitable regenerative braking system, based on the supercapacitor technology, has been studied. Such a system can reduce the electromagnetic stresses of the battery pack increasing the battery life and reducing the maintenance costs (periodic substitutions).

\section{References}

[1]. H. Oman, W. C. Morchin, F. E. Jamerson (1995), Electricbicycle propulsion power, Microelectronics Communications Technology Producing Quality Products Mobile and Portable Power Emerging Technologies, p 555

[2]. W.C. Morchin (1994), Battery-powered electric bicycles, Northcon/94 Conference Record, pp 269 - 274.

[3]. A. Muetze, Y. C. Tan (2005), Performance evaluation of electric bicycles, Industry Applications Conference, Volume 4, pp 2865 - 2872

[4]. E. Starschich, A. Muetze (2007), Comparison of the Performances of Different Geared Brushless-DC Motor Drives for Electric Bicycles, Electric Machines \& Drives Conference IEEE International, Volume 1, pp 140 - 147

[5]. N. Somchaiwong, W. Ponglangka (2006), Regenerative Power Control for Electric Bicycle, SICE-ICASE International Joint Conference, pp 4362-4365 